\title{
Ricerca di soggetti affetti da “iperazotemia familiare non accompagnata da un'insufficienza renale"
}

\author{
Lise Bankir ${ }^{1,2}$, Rosa Vargas-Poussou ${ }^{3-5}$ \\ ${ }^{1}$ Inserm, UMRS 1138, Centre de Recherche des Cordeliers, Paris - France \\ ${ }^{2}$ Université Pierre et Marie Curie, Paris - France \\ ${ }^{3}$ Service de Génétique, Hôpital Européen Georges Pompidou, Paris - France \\ ${ }^{4}$ Inserm, UMR 970, Paris-Centre de Recherche Cardiovasculaire, Paris - France \\ ${ }^{5}$ Centre de Référence des Maladies Rénales Héréditaires de l'Enfant et de l'Adulte (MARHEA), Paris - France
}

\section{Introduzione}

L'iperazotemia familiare non accompagnata da insufficienza renale è caratterizzata da una concentrazione dell'urea plasmatica o ematica di circa 3-5 volte superiore ai valori normali. Tutti gli altri parametri sono nella norma (creatinina plasmatica, filtrazione glomerulare, escrezione urinaria dell'urea ecc.). I soggetti affetti non presentano alcun segno d'insufficienza renale o altri sintomi clinici e ignorano di essere portatori di questa anomalia. Sono generalmente identificati per caso. I loro valori anormali di urea conducono alla ricerca di un'insufficienza renale, che successivamente non viene confermata.

L'ereditarietà dell'iperazotemia familiare sembra seguire un modello autosomico dominante. Due articoli (uno americano e l'altro tedesco) hanno descritto l'azotemia familiare rispettivamente in 5 e 3 soggetti apparentati $(1,2)$, e un terzo articolo (italiano) ha descritto delle forme sporadiche in 14 soggetti non apparentati (3). Un interessante editoriale di cui si raccomanda la lettura accompagnava l'articolo americano (4).

Un aumento isolato del tasso plasmatico d'urea può essere generato da parecchi fattori, ma la causa più probabile potrebbe essere dovuta alla perdita di funzione di una proteina responsabile della secrezione dell'urea nella pars recta del tubulo prossimale (ipotesi proposta da Hays nel suo editoriale del 1978 [4]). L'idea di una secrezione attiva dell'urea nel rene, come la secrezione dell'ippurato, dell'acido urico,

\footnotetext{
Accepted: November 14, 2016

Published online: December 14, 2016

Indirizzo per la corrispondenza:

Dr. Lise Bankir

Inserm, UMRS 1138, Centre de

Recherche des Cordeliers

15 rue de l'École de Médecine

75006 Paris, France

lise.bankir@inserm.fr
}

di nucleotidi ciclici, di xenobiotici, non è attualmente accettata. Eppure, parecchi argomenti basati su osservazioni sperimentali, e l'esistenza della stessa iperazotemia familiare, appoggiano la tesi dell'esistenza di questo processo. Diversi articoli hanno esposto in dettaglio questi argomenti (5-7). Questo meccanismo di trasporto attivo, mediato da una proteina, contribuirebbe a riversare una quantità significativa di urea nell'urina, in più di quella filtrata dai glomeruli (6).

Negli ultimi vent'anni sono state identificate e ampiamente studiate delle proteine mediatrici del trasporto dell'urea nel rene (UT-A1, UT-A2, UT-A3, UT-A4 e UT-B) (8, 9). Questi vettori trasportano l'urea attraverso le membrane cellulari in presenza di un gradiente di concentrazione favorevole e consentono un trasporto $100-1000$ volte più rapido della semplice diffusione. Peraltro, delle analisi funzionali hanno dimostrato l'esistenza di meccanismi di trasporto "attivo" o "secondariamente attivo" dell'urea, mediati dal Na+ e che necessitano di energia, e la cui funzione genera una differenza di concentrazione tra il lume tubulare del nefrone e il sangue circostante. II loro funzionamento è stato descritto nelle branchie degli elasmobranchi, nel rene di certi anfibi, dei ruminanti e dei ratti. I geni che codificano per queste proteine mediatrici del trasporto attivo o secondariamente attivo non sono stati ancora clonati. Un trasportatore attivo è molto probabilmente implicato nella azotemia familiare, come peraltro descritto $(6,7)$.

\section{Proposta di collaborazione}

La nostra équipe è alla ricerca di soggetti che presentano una azotemia familiare (o almeno una iperazotemia persistente) non accompagnata da insufficienza renale, con l'obiettivo di identificare le anomalie molecolari del gene che codifica per la proteina mediatrice della secrezione dell'urea. Malgrado i pochi casi descritti in letteratura, è probabile che questa anomalia non sia poi così rara, dal momento che il più delle volte è ignorata, non essendo accompagnata da sintomi clinici. Tanto più che nella pratica clinica corrente il tasso di urea plasmatica non viene quasi più misurato. 
Con questo articolo ci rivolgiamo ai Nefrologi italiani e sollecitiamo la loro collaborazione per contribuire ad ampliare la conoscenza dei meccanismi molecolari di questa patologia. In pratica noi chiediamo/proponiamo di collaborare segnalandoci i propri pazienti già noti come affetti da questa patologia e restando vigili all'identificazione di nuovi casi.

\section{Disclosures}

Financial support: No financial support was received for this submission.

Conflict of interest: The authors have no conflict of interest.

Questo annuncio è una traduzione di un annuncio pubblicato in francese in: Néphrologie \& Thérapeutique 2016;12:255-256". Tradotta e pubblicata con permesso.

\section{Bibliografia}

1. Hsu CH, Kurtz TW, Massari PU, Ponze SA, Chang BS. Familial azotemia. Impaired urea excretion despite normal renal function. N Engl J Med. 1978;298:117-21.

2. Armsen T, Glossmann V, Weinzierl M, Edel HH. [Familial proximal tubular azotemia. Elevated urea plasma levels in normal kidney function]. Dtsch Med Wochenschr. 1986;111:702-6.

3. Conte G, DalCanton A, Terribile M, et al. Renal handling of urea in subjects with persistent azotemia and normal renal function. Kidney Int. 1987;32:721-7.

4. Hays RM. Familial azotemia. N Engl J Med. 1978;298:160-1.

5. Bankir L, Trinh-Trang-Tan MM. Urea and the kidney. In: Brenner BM, editor. The Kidney (6th Ed). Philadelphia: WB Saunders Company; 2000:637-79.

6. Bankir L, Yang B. New insights into urea and glucose handling by the kidney, and the urine concentrating mechanism. Kidney Int. 2012;81:1179-98.

7. Bankir L. Active urea transport in lower vertebrates and mammals. Subcell Biochem. 2014;73:193-226.

8. Yang B, Bankir L. Urea and urine concentrating ability: new insights from studies in mice. Am J Physiol Renal Physiol. 2005;288:F881-96.

9. Fenton RA. Urea transporters and renal function: lessons from knockout mice. Curr Opin Nephrol Hypertens. 2008;17:513-8. 